[0212-7199 (2006) 23: 5; pp 213-219] ANALES DE MEDICINA INTERNA Copyright $\mathbb{C} 2006$ ARAN EDICIONES, S.L.

AN. MED. INTERnA (Madrid) Vol. 23, N. ${ }^{\circ}$ 5, pp. 213-219, 2006

\title{
Análisis coste-efectividad del uso de atorvastatina en pacientes diabéticos de tipo 2: modelo farmacoeconómico del estudio CARDS
}

\author{
J. FERNÁNDEZ DE BOBADILLA, E. LÓPEZ DE SA', I. ALONSO TRONCOSO², \\ R. MORENO GÓMEZ ${ }^{3}$, C. RUBIO-TERRÉS ${ }^{4}$, J. SOTO ÁLVAREZ \\ Investigación de Resultados en Salud y Farmacoeconomía. Unidad Médica. Pfizer
I+D+i. Madrid. ' $U$ nidad Coronaria. Hospital Gregorio Marañón. Madrid. ${ }^{2}$ Servicio
Endocrinología. Hospital de Montecelo. Pontevedra. ${ }^{3}$ Servicio de Hemodinámica.
Hospital Clínico San Carlos. Madrid. ${ }^{4}$ HERO Consulting. Madrid \\ Investigación de Resultados en Salud y Farmacoeconomía. Unidad Médica. Pfizer
I+D+i. Madrid. ${ }^{1}$ Unidad Coronaria. Hospital Gregorio Marañón. Madrid. ${ }^{2}$ Servicio de
Endocrinología. Hospital de Montecelo. Pontevedra. ${ }^{3}$ Servicio de Hemodinámica.
Hospital Clínico San Carlos. Madrid. ${ }^{4}$ HERO Consulting. Madrid \\ Investigación de Resultados en Salud y Farmacoeconomía. Unidad Médica. Pfizer
I+D+i. Madrid. 'Unidad Coronaria. Hospital Gregorio Marañón. Madrid. ${ }^{2}$ Servicio
Endocrinología. Hospital de Montecelo. Pontevedra. ${ }^{3}$ Servicio de Hemodinámica.
Hospital Clínico San Carlos. Madrid. ${ }^{4}$ HERO Consulting. Madrid \\ Investigación de Resultados en Salud y Farmacoeconomía. Unidad Médica. Pfizer
I+D+i. Madrid. ${ }^{1}$ Unidad Coronaria. Hospital Gregorio Marañón. Madrid. ${ }^{2}$ Servicio
Endocrinología. Hospital de Montecelo. Pontevedra. ${ }^{3}$ Servicio de Hemodinámica.
Hospital Clínico San Carlos. Madrid. ${ }^{4}$ HERO Consulting. Madrid
}

COST-EFFECTIVENESS ANALYSIS OF THE USE OF ATORVASTATIN IN PATIENTS WITH TYPE 2 DIABETES MELLITUS: A PHARMACOECONOMIC MODEL OF THE CARDS STUDY

\section{RESUMEN}

Fundamento y objetivo: Efectuar un análisis coste-efectividad del uso de Atorvastatina $10 \mathrm{mg}$ en la prevención primaria de la enfermedad cardiovascular en el paciente con Diabetes Mellitus tipo 2 (DM2).

Método: Se elaboró un modelo retrospectivo y determinístico basado en un análisis de decisión a partir del estudio CARDS (Collaborative Atorvastatin Diabetes Study). En el estudio CARDS se había demostrado que Atorvastatina $10 \mathrm{mg}$ versus placebo en prevención primaria en pacientes con DM2 y alguna patología asociada, reduce la morbimortalidad cardiovascular $(5,8 v s .9,0 \%, p=0,001)$. En el presente análisis coste-efectividad, las unidades de efectividad utilizadas fueron años de vida ganados (AVG) y años de vida ajustados por calidad (AVAC), obtenidos a partir de las diferencias de morbimortalidad y de la esperanza de vida de los diabéticos, con y sin eventos previos, obtenidos de la literatura. El consumo de recursos de las alternativas en evaluación se ha extraído del estudio CARDS aplicando costes españoles.

Resultados: El cociente coste-efectividad incremental derivado de utilizar Atorvastatina $10 \mathrm{mg}$ versus placebo fue $5.886 €$ por AVG y de $8.046 €$ por AVAC. Los análisis de sensibilidad confirmaron la estabilidad del modelo.

Conclusiones: En la prevención primaria del riesgo cardiovascular en pacientes diabéticos tipo 2, el uso de Atorvastatina $10 \mathrm{mg}$ es coste-efectivo, con un coste por AVG y por AVAC por debajo de otras alternativas empleadas ampliamente en el Sistema Nacional de Salud español, y también por debajo de un valor que podría considerarse como un umbral razonable para nuestro país, situado oficiosamente en torno a los $30.000 €$ por AVAC.

PALABRAS CLAVE: Diabetes mellitus. Prevención primaria. Coste efectividad. Atorvastatina.

\section{ABSTRACT}

Background and objective: To perform a cost-effectiveness analysis of the use of Atorvastatin $10 \mathrm{mg}$ in the primary prevention of cardiovascular disease in patients with type 2 diabetes (DM2).

Method: A deterministic and retrospective model by a decision analysis based on CARDS study (Collaborative Atorvastatin Diabetes Study) was performed. In the CARDS study, a significant reduction in cardiovascular morbimortality by the use of Atorvastatin $10 \mathrm{mg}$ versus placebo (5.8 vs. 9.0\%, $p=0.001)$ in DM2 patients with an additional condition, had previously been demonstrated. In the present cost-effectiveness analysis, effectiveness units were life years gained ( $L Y G)$ and quality adjusted life years (QALY), obtained from differences in morbimortality and life expectancy in DM2 patients, with and without previous cardiovascular events. Costs of the evaluated alternatives were obtained from the CARDS results.

Results: Incremental cost-effectiveness ratio of using Atorvastin 10 $m g$ versus placebo was 5,886 € per $L Y G$ and 8,046 € per QALY. Sensitivity analyses confirmed the model stability.

Conclusions: In the primary prevention of the cardiovascular disease in type 2 diabetic patients, the use of Atorvastatin $10 \mathrm{mg}$ is costeffective, with a cost per $L Y G$ and per QALY below that of other alternatives widely used in the Spanish National Health System, and also below a value considered as a reasonable threshold for our country, which might unofficialy be around $30,000 € / Q A L Y$.

KEY WORDS: Diabetes mellitus. Primary prevention. Cost effectiveness. Atorvastatin.

Fernández de Bobadilla J, López de Sa E, Alonso Troncoso I, Moreno Gómez, R, Rubio-Terrés C, Soto Álvarez. J. Análisis coste-efectividad del uso de Atorvastatina en pacientes diabéticos de tipo 2: modelo farmacoeconómico del estudio CARDS. An Med Interna (Madrid) 2006; 23: 213-219.

Fuente de financiación: estudio efectuado con una ayuda a la investigación de Pfizer, S.A.

Resultados preliminares de este trabajo han sido presentados en el 47 Congreso de la Sociedad Española de Endocrinología y Nutrición. Madrid, Mayo de 2005.

\section{INTRODUCCIÓN}

La diabetes está transformándose en un problema de dimensiones epidémicas. Se calcula que en poco más de 20 años la cantidad de personas afectadas por dicha enfermedad crecerá aproximadamente un $120 \%$ en todo el planeta, fenómeno que se estima habrá de ser más marcado en los países en 
desarrollo. Las implicaciones sociales de este panorama se resumen en el hecho de que la enfermedad cardiovascular (ECV) y especialmente la enfermedad coronaria es la principal causa de morbi-mortalidad entre los pacientes diabéticos. Asociado al aumento de la prevalencia de la diabetes, inevitablemente se va a producir un aumento en las proporciones de morbilidad y mortalidad relacionadas con la ECV.

En España la prevalencia de la diabetes mellitus tipo 2 (DM2) es también un importante problema de salud pública. En particular, la prevalencia de la diabetes en adultos de mediana edad oscila entre el 7 y el $16 \%$ en función del área geográfica y de los criterios diagnósticos utilizados $(1,2)$. La DM2 es una de las principales causas de muerte en la mayoría de los países desarrollados. En España, supone la tercera causa de muerte entre las mujeres y la séptima entre los varones (3). Los pacientes diabéticos presentan 2 a 4 veces más riesgo que la población general de desarrollar enfermedad cardiovascular (4), así como más riesgo de presentar una enfermedad microvascular específica (5-7).

Un estudio más reciente, el CODE-2 proporcionó el primer intento coordinado para evaluar el coste total asociado al tratamiento de pacientes con DM2 en países europeos. El coste anual sanitario medio por paciente en el estudio fue de $1.305,15$ euros, incluyendo los motivos relacionados tanto con el control de la diabetes como con sus complicaciones. Los pacientes sin complicaciones originaron sólo el 13,7\% del gasto total, mientras que los que presentaban algún tipo de complicación (micro, macrovascular o ambas) supusieron el $64,5 \%$. Las complicaciones cardiovasculares son las principales responsables del coste de la diabetes. Cuando éstas aparecen, se duplica el coste global y se cuadruplica el de las hospitalizaciones, por lo que es necesario establecer medidas de control estrictas para reducir su incidencia y poder actuar en el campo de la prevención secundaria (8). En otro estudio que dividía el coste por categorías, se demostró que los pacientes diabéticos tuvieron una media de ingresos hospitalarios superior a los no diabéticos (9).

Las complicaciones macroangiopáticas, especialmente la enfermedad arterial coronaria y el ictus cerebral, son responsables de casi el $80 \%$ de la mortalidad y del $75 \%$ de las hospitalizaciones en los pacientes diabéticos (10). Las estatinas se han mostrado eficaces en la prevención secundaria (4S, CARE, LIPID, GREACE, HPS, MIRACL, TNT y PROVE-it) y primaria (WOSCOPS, CARDS y ASCOT) de las enfermedades cardiovasculares. Este beneficio incluye también a la población de edad avanzada (HPS, PROSPER, CARDS, ASCOT) y se mantiene incluso para los rangos de LDL-C basal bajos (HPS, PROVE-IT, CARDS y TNT). Recientemente, se ha demostrado que el tratamiento con estatinas puede disminuir la incidencia de estos eventos cardiovasculares cuando se utilizan para la prevención primaria en pacientes con DM2 (11,12). En el estudio CARDS (Collaborative Atorvastatin Diabetes Study), un ensayo clínico multicéntrico, aleatorizado, controlado con placebo, doble ciego, que incluyó 2.838 pacientes (1.428 tratados con Atorvastatina $10 \mathrm{mg} \mathrm{y}$ 1.410 con placebo) se ha demostrado que Atorvastatina $10 \mathrm{mg}$ es segura y eficaz para reducir el riesgo de primeros episodios de enfermedad cardiovascular, incluyendo el ictus, en los pacientes con DM2 con alguna patología asociada (retinopatía, micro o macroalbuminuria, tabaquismo o hipertensión) y que no presentan un valor elevado de LDL-C (LDL-Colesterol basal $\leq 160 \mathrm{mg} / \mathrm{dl}$ ) (12).
IMPORTANCIA DE LA FARMACOECONOMÍA EN LA ASIGNACIÓN DE RECURSOS

Los recursos son limitados y el coste sanitario continúa aumentando en España y en los países de nuestro entorno. Los estudios farmacoeconómicos, son herramientas que ayudan a la toma de decisiones en la asignación de los recursos. Es cierto que tienen limitaciones, pero también que no recurrir a la farmacoeconomía, supone la renuncia a que los aspectos técnicos más objetivos tengan peso a la hora de configurar el criterio utilizado en la asignación de recursos.

Para el clínico, las evaluaciones económicas resultan difíciles de interpretar y, en ocasiones, pueden generar escepticismo. Esto se debe en gran parte a que la farmacoeconomía no es estrictamente una ciencia que produce conocimiento utilizando el método científico. Es más una disciplina, que participa de ciencias biológicas, económicas y sociales y cuya aplicación práctica tiene, probablemente, más que ver con la ayuda a la toma de decisiones relacionadas con la asignación de recursos que con la producción de conocimiento.

El primer paso para poder decidir con criterio si una estrategia de tratamiento es coste-efectiva, es disponer del resultado en unidades que sean comparables entre diferentes áreas terapéuticas. La relación coste/efecto, se presenta en unidades monetarias (€)/unidades de efectividad; pero las unidades de efectividad pueden ser desde infarto agudo de miocardio evitado, a mm de $\mathrm{Hg}$ de presión arterial o años de vida ganados. Las únicas unidades de efectos que resultan directamente comparables entre áreas terapéuticas son los años de vida ganados (AVG) y los años de vida ajustados por calidad (AVAC). Estas son, por tanto, las únicas unidades en las que existen intentos de establecer un límite generalizable que determine cuando una estrategia es eficiente o coste/efectiva.

El concepto de AVAC merece una explicación introductoria. Suponiendo que la calidad de vida relacionada con la salud fluctúa entre 0 (la muerte) y 1 (la salud perfecta). Un AVAC sería el equivalente a un año de vida vivido con una salud perfecta; pero igualmente un AVAC podría obtenerse de más de una año de vida con una salud no perfecta, por ejemplo, de 2 años vividos con una salud de 0,5 . La ventaja del $\mathrm{AVAC}$ es que permite comparar entre áreas muy diversas e integrar en una sola unidad cantidad y calidad de vida.

\section{OBJETIVO}

El objetivo de este estudio es un análisis coste-efectividad del uso de Atorvastatina en la prevención primaria de la enfermedad cardiovascular en el paciente con DM2.

\section{MATERIAL Y MÉTODOS}

\section{POBLACIÓN DIANA}

La población diana fueron los pacientes incluidos en el estudio CARDS: sujetos con DM2, de una edad entre 40 y 75 años, con niveles de LDL-colesterol $<160 \mathrm{mg} / \mathrm{dl}$ y niveles de triglicéridos $\leq 600 \mathrm{mg} / \mathrm{dl}$ y al menos una de las siguientes patologías asociadas: hipertensión arterial, retinopatía, microalbuminuria, macroalbuminuria y tabaquismo (12). 


\section{MODELO FARMACOECONÓMICO Y ESTIMACIÓN DE LA EFECTIVIDAD}

El estudio consistió en un modelo farmacoeconómico, entendido como un esquema teórico que permite hacer simulaciones de procesos sanitarios complejos relacionados con medicamentos y que es elaborado, siguiendo un protocolo previamente establecido, mediante estimaciones obtenidas a partir de los datos disponibles de eficacia, toxicidad y costes de las alternativas comparadas (13). Para ello, se hizo un análisis de decisiones determinístico y retrospectivo, con una estructura que se representa en la figura 1 . Se consideró que un paciente con DM2 podría o no padecer eventos cardiovasculares (IAM mortal o no, muerte coronaria debida a otras causas, angina inestable, muerte súbita resucitada, revascularización coronaria e ictus mortal o no) con diferentes probabilidades, derivadas del estudio CARDS: la morbimortalidad cardiovascular se produjo en un $5,8 \%$ de los pacientes, después de 3,9 años de tratamiento con Atorvastatina $10 \mathrm{mg}$ (83 eventos en 1.418 pacientes) y en el $9,0 \%$ de los pacientes controlados con placebo (127 eventos en 1.410 pacientes). Las probabilidades de los diferentes eventos se indican en la tabla I.

La efectividad se midió como años de vida ganados (AVG) y años de vida ajustados por su calidad (AVAC) gana-

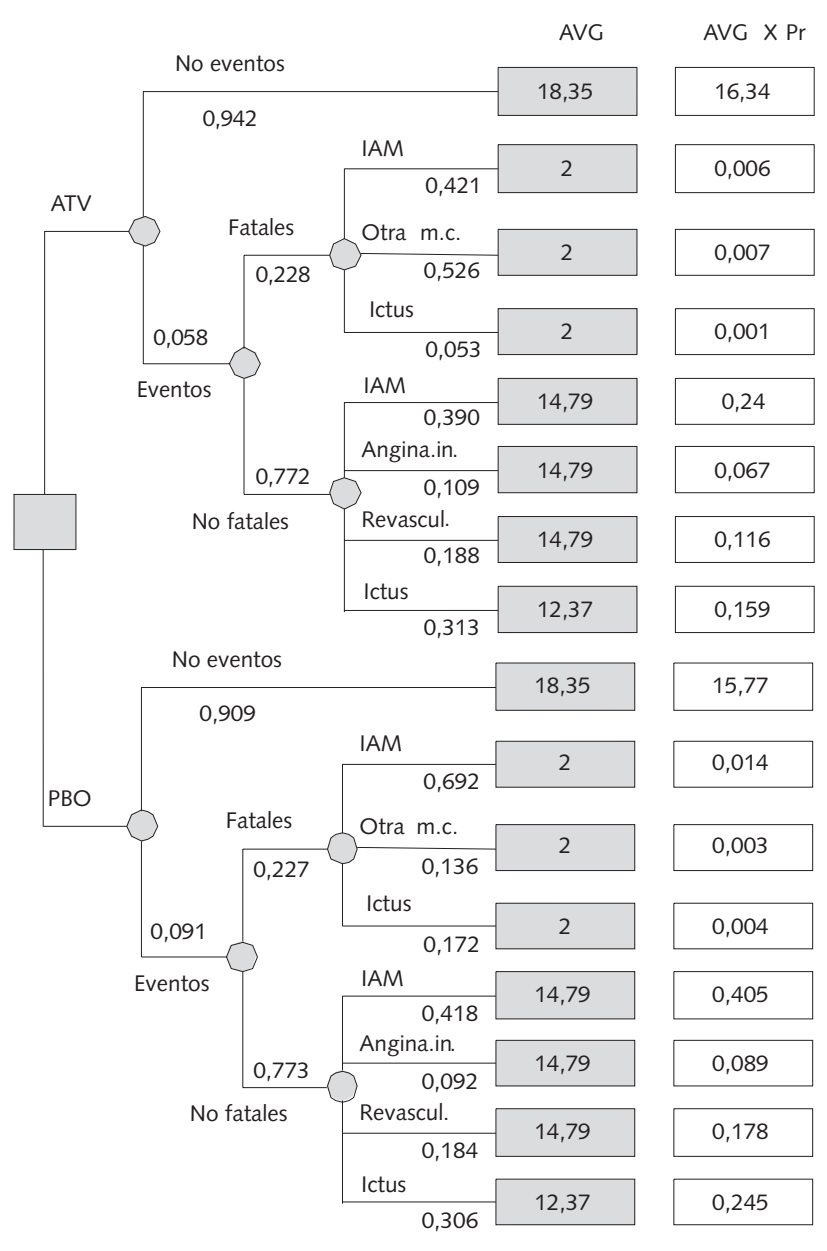

Fig. 1.
TABLA I

PROBABILIDADES DE LOS EVENTOS CARDIOVASCULARES OBSERVADAS EN EL ESTUDIO CARDS (12)

\begin{tabular}{lcc}
\hline Item & Atorvastatina & Placebo \\
\hline Morbimortalidad cardiovascular total & 0,058 & 0,090 \\
\hline Infarto agudo de miocardio mortal & 0,096 & 0,157 \\
Infarto agudo de miocardio no mortal & 0,301 & 0,323 \\
Otras muertes coronarias & 0,120 & 0,031 \\
Angina inestable & 0,084 & 0,071 \\
Muerte súbita resucitada & 0,000 & 0,000 \\
Revascularización coronaria & 0,145 & 0,142 \\
Ictus mortal & 0,012 & 0,039 \\
Ictus no mortal & 0,242 & 0,237 \\
\hline
\end{tabular}

dos en los pacientes tratados con Atorvastatina $10 \mathrm{mg}$, en comparación con los no tratados. Los AVG se estimaron a partir de los pacientes del estudio CARDS que completaron el seguimiento (1.421 pacientes en el grupo de Atorvastatina 10 mg y 1.398 en el grupo placebo).

La esperanza de vida considerada en los pacientes diabéticos fue la observada en el estudio de Ontario (a la edad de 63,5 años fue de 12,29 y de 13,84 años para hombres y mujeres, respectivamente) (14). Basándose en un modelo fundamentado en el UKPDS, se estimó que haber sufrido un infarto disminuía la calidad de vida del paciente diabético en un 5,5\% y haber sufrido un ictus en un 10,8\% (15). A partir de estos valores, se estimó que la esperanza de vida ajustada o no por calidad, ponderada para ambos sexos, sería de 12,78 y 16,35 años, respectivamente. Por otra parte, la esperanza de vida en los pacientes con DM2 que han sufrido un infarto de miocardio o un ictus cerebral, se obtuvo a partir de los resultados del análisis de supervivencia de Caro y cols. (16). El horizonte temporal de la simulación fue de 4 años, aproximadamente la duración promedio del estudio CARDS.

El estudio se hizo desde la perspectiva del Sistema Nacional de Salud (SNS) español, por tanto considerando únicamente los costes directos sanitarios.

Se siguieron las directrices generales para la realización de análisis farmacoeconómicos en España (17), así como las directrices publicadas por la Oficina Canadiense de Coordinación de la Evaluación de las Tecnologías Sanitarias (18) y los Principios de buenas prácticas de modelización de la International Society for Pharmacoeconomics and Outcomes Rese$\operatorname{arch}$ (ISPOR) (19).

Finalmente, se hizo un descuento anual del 3\% para los costes y la efectividad (AVAC, AVG). Dado que los costes y efectos ocurren en el futuro, hay que convertirlos a valor presente. A tal fin se utiliza la tasa de descuento. El descuento del $3 \%$ a costes y efectos es estándar.

\section{ESTIMACIÓN DE LOS COSTES}

La estimación de los costes de una enfermedad tratada con un determinado fármaco se hace mediante la identificación y cuantificación de los recursos sanitarios que conlleva y asignando a los recursos unos determinados costes unitarios. De 
este modo, se estimaron los costes medios para un paciente de tipo CARDS tratado con Atorvastatina $10 \mathrm{mg}$ en la prevención primaria cardiovascular, desde la perspectiva del SNS, considerándose dos tipos de coste: el de adquisición de Atorvastatina $10 \mathrm{mg}$, obtenido de la base de datos de Portalfarma (20) y el coste de los eventos cardiovasculares, tomados de una base de datos de costes sanitarios españoles (21) a través del coste unitario de los distintos grupos relacionados con el diagnóstico (GRDs). A este respecto, se hicieron varias asunciones: a) que el coste de la parada cardiaca resucitada fuera similar al de la parada de causa desconocida; b) que el coste del ictus mortal fuera similar, siendo conservadores, al de la parada cardiaca de causa desconocida; c) que el coste de la muerte coronaria no debida a IAM fuera similar al de la muerte coronaria por IAM; y d) que todos los procedimientos de revascularización coronaria fueran no quirúrgicos (angioplastia) y con cateterismo diagnóstico previo, ante la ausencia de este último dato. No se consideró el coste de las reacciones adversas, debido a que en el estudio CARDS no se observaron diferencias significativas en la frecuencia de acontecimientos adversos graves $(1,1 \%)$ entre Atorvastatina $10 \mathrm{mg}$ (19 casos) y placebo (20 casos).

Los costes unitarios de los recursos sanitarios considerados en el análisis, expresados en euros $(€)$ de 2.004, se recogen en la tabla II.

\section{CASO BÁSICO Y ANÁLISIS DE SENSIBILIDAD}

En el caso básico del modelo se utilizaron los valores promedio de los costes de los eventos cardiovasculares. Se hicieron los siguientes análisis de sensibilidad: a) probabilísticos de Monte Carlo de primer orden (22); b) simples univariantes utilizando los costes mínimos y máximos de los eventos; y c) considerando el cumplimiento medio real del tratamiento con Atorvastatina $10 \mathrm{mg}$ durante 4 años en los pacientes del estudio CARDS $(85 \%)$.

\section{PROGRAMA INFORMÁTICO}

El análisis del modelo se hizo mediante el programa DATA 3.5 for Healthcare de TreeAge.
TABLA II

COSTES UNITARIOS ASIGNADOS A LOS RECURSOS EN EL ANÁLISIS FARMACOECONÓMICO

\begin{tabular}{lll}
\hline Recurso (n ${ }^{\circ}$ tipo) & $\begin{array}{l}\text { Coste unitario }(€) \\
\text { (mínimo-máximo) }\end{array}$ & Referencia \\
\hline $\begin{array}{lll}\text { Atorvastatina } \\
\text { Cardyl (28 comprimidos de 10 mg)* }\end{array}$ & 27,85 & 17 \\
& & \\
Eventos cardiovasculares & $4953,69(1413,68-8553,73)$ & 18 \\
Infarto agudo de miocardio mortal & $5278,70(3508,49-8124,49)$ & 18 \\
Infarto agudo de miocardio no mortal & 4953,69 & 18 \\
Otras muertes coronarias & $2342,91(2226,34-2459,48)$ & 18 \\
Angina inestable & $2771,52(1742,59-5546,81)$ & 18 \\
Muerte súbita resucitada & 8575,23 & 18 \\
Revascularización coronaria & 2771,52 & 18 \\
Ictus mortal & 4227,67 & 18 \\
Ictus no mortal &
\end{tabular}

* Precio de venta al público + 4\% de IVA.

\section{RESULTADOS}

\section{CASO BÁSICO}

Las consecuencias económicas de los resultados del estudio CARDS utilizando datos de costes españoles muestran que el tratamiento con $10 \mathrm{mg}$ de Atorvastatina en pacientes con diabetes mellitus tipo 2 supone unos costes medios de tratamiento por paciente, mayores que la opción de no tratar, $1.573,81 €$ con Atorvastatina $10 \mathrm{mg} v \mathrm{~s}$. $455,41 €$ con placebo, con un incremento en el coste total en el grupo de Atorvastatina $10 \mathrm{mg}$ de $1.118,4 €$ durante el periodo del estudio, equivalentes a $279,6 €$ adicionales por paciente y año. Este incremento se ve parcialmente compensado por el mayor número de eventos evitados con Atorvastatina $10 \mathrm{mg}$ y por tanto el menor coste asociado a complicaciones cardiovasculares.

Basándonos en el beneficio a largo plazo, el tratamiento con $10 \mathrm{mg}$ de Atorvastatina durante 4 años origina, en comparación con la opción de no administrar un tratamiento para la prevención primaria cardiovascular, la ganancia de 0,19 años de vida y de 0,139 AVAC por cada paciente tratado. Esta mayor eficacia tendría un coste adicional de $1.118 €$ por paciente (Tabla III).

TABLA III

RESULTADOS DEL ANÁLISIS FARMACOECONÓMICO DE ATORVASTATINA EN PACIENTES CON DIABETES MELLITUS DE TIPO 2. CASO BÁSICO

\begin{tabular}{lccccc}
\hline Tratamiento & Costes $(€)$ & Diferencia de costes $(€)$ & Efectividad (AV) & $\begin{array}{c}\text { Diferencia de } \\
\text { efectividad (AVG) }\end{array}$ & $\begin{array}{c}\text { Coste- efectividad } \\
\text { incremental (€/AVG) }\end{array}$ \\
\hline Atorvastatina & $1.573,81$ & $1.118,40$ & 14,189 & 0,190 & $5.886,31$ \\
Placebo & 455,41 & & 13,999 & & \\
\hline Tratamiento & Costes $(€)$ & Diferencia de costes (€) & Efectividad (AVAC) & $\begin{array}{c}\text { Diferencia de } \\
\text { efectividad (AVAC) }\end{array}$ & $\begin{array}{c}\text { Coste- efectividad } \\
\text { incremental (€/AVAC ganado) }\end{array}$ \\
\hline Atorvastatina & $1.573,81$ & $1.118,40$ & 11,114 & 0,139 & $8.046,04$ \\
Placebo & 455,41 & & 10,975 & & \\
\hline
\end{tabular}

Abreviaturas: AV: año de vida; AVAC: año de vida ajustado por su calidad; AVG: año de vida ganado. 
El coste de ganar un año de vida o un AVAC con Atorvastatina $10 \mathrm{mg}$, en comparación con placebo, ascendería a 5.886 $€$ y $8.046 €$, respectivamente y para una tasa de descuento del 3\% (Tabla III).

\section{ANÁLISIS DE SENSIBILIDAD}

El resultado del caso básico fue estable en los análisis de sensibilidad, con resultados que oscilaron entre 4.874 y 6.500 $€$ por AVG y entre 6.663 y $8.944 €$ por cada AVAC adicional (Tabla IV). El menor valor de coste-efectividad se observó, como era previsible, cuando se consideró el cumplimiento terapéutico del $85 \%$ habido en el estudio CARDS, en lugar del $100 \%$ teórico del caso básico del análisis.

\begin{tabular}{lcc}
\multicolumn{4}{c}{ TABLA IV } \\
\multicolumn{3}{c}{$\begin{array}{c}\text { RESULTADOS DEL ANÁLISIS DE SENSIBILIDAD. COSTE- } \\
\text { EFECTIVIDAD INCREMENTAL }\end{array}$} \\
\hline Escenario & Coste/AVG (€) & $\begin{array}{c}\text { Coste/AVAC } \\
\text { ganado (€) }\end{array}$ \\
\hline Caso básico & $8.046,04$ \\
Monte Carlo & $5.886,31$ & $8.944,56$ \\
Costes mínimos* $^{*}$ Costes máximos* & $6.500,41$ & $8.285,90$ \\
Cumplimiento CARDS $^{*}$ & $6.061,79$ & $7.722,37$ \\
\hline
\end{tabular}

*De los eventos cardiovasculares. Abreviaturas: AVAC: año de vida ajustado por su calidad; AVG: año de vida ganado.

\section{DISCUSIÓN}

\section{INTERPRETACIÓN GENERAL, DE LOS RESULTADOS DE COSTE- EFECTIVIDAD}

Los recursos económicos disponibles son limitados y el coste sanitario continúa aumentando en España y en los países de nuestro entorno, con lo cual existe una necesidad de optimizar la eficiencia (análisis de la relación entre los recursos consumidos o costes y los resultados obtenidos) de la utilización de los recursos $(23,24)$. Se está intentando encontrar un criterio de eficiencia generalizable que permita a los decisores sanitarios optimizar la eficiencia (25).

Las opciones más coste-efectivas no tienen por qué ser las más baratas ni las más efectivas. Sin embargo, a menudo se decide financiar una tecnología sanitaria exclusivamente porque ahorra dinero, olvidando que una intervención también será eficiente si el beneficio extra que produce compensa su coste adicional (26). En realidad esta sería una decisión de tipo financiero (que sólo tiene en cuenta los costes), pero no farmacoeconómica, que por definición, también debe tener en cuenta los efectos. Cuando una alternativa terapéutica es a la vez más efectiva y más barata que otra, la decisión es evidente. El problema surge cuando se evalúan medicamentos o tecnologías que producen mejores resultados clínicos pero con costes asociados más elevados, es entonces cuando hay que definir cuándo un beneficio extra compensa su coste adicional. Para ello es necesario decidir si el valor del coste extra necesario para conseguir una unidad adicional de resultado clínico (correspondiente al cociente coste efectividad incremental o CCEI) resulta socialmente aceptable. Ese es, precisamente, el umbral que los agentes decisores y la sociedad tendrán que fijar para cada país. Las estrategias terapéuticas con un CCEI por debajo del fijado automáticamente se financiarían y las que se encuentren por encima de ese valor tendrán que ser sometidas a criterios adicionales de evaluación antes de decidir su financiación.

Ya existen algunos países donde se recomienda un valor umbral. Así, en Estados Unidos, una intervención sanitaria presenta una relación coste-efectividad aceptable si el coste adicional de cada AVAC ganado es inferior a 50.000 dólares e inaceptable cuando supera los 100.000 dólares por AVAC. En Europa los valores oscilan dependiendo del país. En el Reino Unido están entre 33.000 y 50.000 euros; en Holanda entre 20.000 y 30.000 euros; en Francia entre 24.000 y 36.000 euros; en Alemania entre 25.000 y 37.000 euros y en Italia entre 21.000 y 31.000 euros.

Estos límites se establecieron de una forma heterogénea y arbitraria: 1) a partir de recomendaciones efectuadas por los autores de los estudios farmacoeconómicos publicados (27), 2) por aproximación al coste de técnicas cuya financiación nadie pone en cuestión (como la diálisis en los pacientes con insuficiencia renal terminal); o 3) por representar los valores de las tecnologías usualmente recomendadas por agencias de evaluación como el NICE.

En nuestro país este es un tema aún no resuelto. En una revisión publicada en el año 2002, los autores hicieron una propuesta para el rango de valor umbral del CCEI para que un nuevo medicamento pueda ser considerado coste-efectivo. El límite de coste-efectividad establecido fue oficiosamente de 30.000 euros por AVG (28). Este artículo ha tenido cierta repercusión en España y, actualmente, se acepta de forma orientativa que una intervención sanitaria podría considerarse eficiente cuando el coste por AVAC es inferior a los 30.000 euros. Esto equivale aproximadamente a 25.000 euros por AVG en pacientes de este tipo. Esta cifra no es, en absoluto, un valor oficial ni aceptado por todos pero, a falta de otro criterio puede ayudar a poner en perspectiva los valores del cociente coste-efectividad incremental.

\section{IMPORTANCIA CLÍNICA DE ATORVASTATINA $10 \mathrm{mg}$ EN EL PACIENTE DIABÉTICO}

En el estudio CARDS, Atorvastatina $10 \mathrm{mg}$ mostró una reducción de riesgo del 37\% para el end-point primario formado por el conjunto de: muerte de origen cardiovascular, IAM, angina inestable que requiere rehospitalización, parada cardiaca, revascularización e ictus. La reducción del riesgo de ictus fue de un $48 \%$ en los pacientes en tratamiento con Atorvastatina $10 \mathrm{mg}$. La reducción de la mortalidad total del grupo tratado con Atorvastatina $10 \mathrm{mg}$ fue del $27 \%$. Las diferencias encontradas fueron estadísticamente significativos para las dos variables anteriores, y hubo una tendencia estadística para la una mortalidad total $(\mathrm{p}=0,059)$ con Atorvastatina $10 \mathrm{mg}$. Los resultados sobre la reducción de los eventos cardiovasculares fueron consistentes con independencia de los niveles basales de lípidos, edad, sexo y otros factores de riesgo. Ambos brazos de tratamiento fueron seguros y bien tolerados. El estudio CARDS demuestra que el tratamiento con Atorvas- 
tatina $10 \mathrm{mg}$ es seguro y eficaz en disminuir la aparición del primer evento cardiovascular (incluyendo el ictus) en diabéticos tipo 2 con niveles de lípidos normales (c-LDL $160 \mathrm{mg} / \mathrm{dl}$ ). Asimismo, el CARDS sugiere que no hay justificación para que exista un nivel determinado de c-LDL a partir del cual los pacientes con DM2 deban iniciar tratamiento con estatinas y subrayan la atención que debe prestarse al control de los lípidos, además de la glucemia y la presión arterial en pacientes con DM2. Esto plantea la cuestión clínica práctica de si existen pacientes con DM2 con riesgo suficientemente bajo como para no precisar tratamiento.

\section{INTERPRETACIÓN DE LA EVALUACIÓN ECONÓMICA BASADA EN EL ESTUDIO CARDS}

De acuerdo con los resultados del modelo, los pacientes con DM2 sometidos a prevención primaria de eventos cardiovasculares con Atorvastatina $10 \mathrm{mg}$, obtienen más AVG y más AVAC que los pacientes sin tratamiento preventivo.

En la interpretación de esta evaluación económica debemos considerar que se trata de un modelo teórico (que es, por definición, una simulación simplificada de la realidad) basado en los resultados de un ensayo clínico, por lo que sus resultados deben considerarse como estimaciones para pacientes del tipo de los incluidos en el estudio CARDS, que pueden ser útiles como una herramienta para la toma de decisiones clínicas.

Según los resultados del modelo, el uso de Atorvastatina $10 \mathrm{mg}$ en prevención primaria del riesgo cardiovascular en pacientes diabéticos de tipo 2 es coste-efectivo, con un coste por AVG y por AVAC ganado muy por debajo de $30.000 €$ (28), una cifra que en nuestro país puede servir de umbral oficioso orientativo para considerar una alternativa como eficiente.

\section{LIMITACIONES Y FORTALEZAS DE LA EVALUACIÓN ECONÓMICA}

Dentro de la farmacoeconomía, con frecuencia debemos recurrir a los modelos, cuando las preguntas no pueden contestarse directamente a través de datos de un estudio. En esta evaluación económica, se recurre a un modelo para obtener datos que permitan transformar las unidades de eficacia disponibles, AVG o AVA C. En el estudio CARDS se evalúan, entre otras, muerte, infarto agudo de miocardio e ictus. El número de años de vida ganados que representa evitar una muerte, dependerá de la expectativa de vida promedio de la población estudiada. Del mismo modo, el número de años de vida ganados que representa evitar un infarto agudo de miocardio (IAM), dependerá de cuánto acorta la expectativa de vida tener un IAM a una edad determinada. Para hacer esta transformación de eventos a AVG y AVACs, es necesario, por tanto, aplicar un modelo. Aunque sabemos que los modelos tienen limitaciones, en ocasiones como las del presente estudio son la única alternativa disponible para tener los resultados en unidades interpretables.

Una limitación específica de este estudio consiste en que, al no disponerse de datos españoles, los AVAC se estimaron a partir de un trabajo canadiense, que recogió la calidad de vida en pacientes diabéticos tras sufrir eventos cardiovasculares. Probablemente, como consecuencia de esto, tienen mayor valor los datos de coste por año de vida ganado. Al mismo tiempo, deben señalarse como "fortalezas" del modelo que la estimación de las probabilidades de transición se hizo a partir de los resultados de un ensayo clínico aleatorizado y que la estimación de los costes unitarios se hizo a partir de una base de datos de costes españoles.

Para intentar minimizar las limitaciones del modelo, en el caso básico se tomaron supuestos conservadores (y promedios) y se hicieron análisis de sensibilidad probabilísticos y considerando varios escenarios extremos, confirmándose la estabilidad del caso básico del análisis.

\section{CONCLUSIONES}

El estudio CARDS no solo demuestra que el tratamiento con Atorvastatina $10 \mathrm{mg}$ es un tratamiento seguro y eficaz para disminuir la aparición del primer evento cardiovascular en diabéticos tipo 2 con niveles de colesterol $\mathrm{LDL}<160$ $\mathrm{mg} / \mathrm{dl}$; sino que lo hace a un coste muy aceptable. El coste de ganar un año de vida o un AVAC con Atorvastatina $10 \mathrm{mg}$, en comparación con placebo, fue de $5.886 €$ y $8.046 €$, respectivamente. Por tanto, en la prevención primaria del riesgo cardiovascular en pacientes diabéticos de tipo 2, el uso de Atorvastatina es coste-efectivo, con un coste por AVG y por AVAC por debajo de otras alternativas empleadas ampliamente en el SNS y de un valor que podría considerarse como un umbral razonable para nuestro país, que podría situarse oficiosamente en torno a los $30.000 €$ por AVAC (28).

\section{Bibliografía}

1. Arroyo J, Badía X, Calle H. Tratamiento de los pacientes con diabetes mellitus tipo 2 en atención primaria en España. Med Clin (Barc) 2005; 125: $166-72$.

2. Goday A, Gabriel R, Ascaso JF, et al. Despistaje y seguimiento de la insulina resistencia en España: Estudio DESIRE. Avances en Diabetología, 2004. Sociedad Española de Diabetes. Vol.20.Supl.1. Mayo 2004.

3. Goday A, Serrano Ríos M. Epidemiología de la Diabetes Mellitus en España. Revisión crítica y nuevas perspectivas. Med Clin (Barc) 1994; 102: 306-315.

4. Gimeno Orna JA, Boned Juliani B, Lou Arnal L. M, et al. Mortalidad en una cohorte de pacientes con diabetes tipo 2 del Área de Alcañiz. An
Med Interna (Madrid) 2002; 19: 14-18.

5. Cerveny JD, Leder RD, Weart CW Issues surrounding tight glycemic control in people with type 2 diabetes mellitus.Ann Pharmacother 1998; 32: 896-905

6. Calvo Romero, J. M $\mathbf{M}^{\mathrm{a}}$ and Lima Rodríguez, E. M $\mathbf{M}^{\mathrm{a}}$ Objetivos y estrategia terapéutica en la diabetes mellitus tipo 2 . An Med Interna (Madrid) 2001; 18: 41-48.

7. Pérez García, R., Rodríguez Benítez, P., Dall’Anesse, C. et al. Preocupante incremento de la diabetes como causa de insuficiencia renal terminal: Evaluación del tratamiento sustitutivo. An Med Interna (Madrid) 2001; 18: 7-14. 
8. Williams R, Van Gaal L, Lucioni C, CODE-2 Advisory Board. Assessing the impact of complications on the costs of Type II diabetes. Diabetologia 2002; 45: S13-S17.

9. Pascual JM.,González, C, Juan García, S. de. Impacto de la diabetes mellitus en los costes de hospitalización. Med Clin (Barc), 1996; 107.

10. National Diabetes Data Group. Diabetes in America. National Institutes of Health. National Institute of Diabetes and Digestive and Kidney Diseases. NIH Publication No. 95-1468. Bethesda: NIH, 1995.

11. Collins R, Armitage J, Parish S, Sleigh P, Peto R; Heart Protection Study Collaborative Group. MRC/BHF Heart Protection Study of cholesterol-lowering with simvastatin in 5963 people with diabetes: a randomised placebo-controlled trial. Lancet 2003; 361: 2005-16.

12. Colhoun HM, Betteridge JD, Durrington PN, et al. Primary prevention of cardiovascular disease with atorvastatin in type 2 diabetes in the Collaborative Atorvastatin Diabetes Study (CARDS): multicentre randomised placebo-controlled. Lancet 2004; 364: 685-96.

13. Rubio-Terrés C, Sacristán JA, Badía X, Cobo E, García Alonso F, por el Grupo ECOMED. Métodos utilizados para realizar evaluaciones económicas de intervenciones sanitarias. Med Clin (Barc) 2004; 122: 578-83.

14. Manuel DG, Schultz SE. Health-related quality of life and health-adjusted life expectancy of people with diabetes in Ontario, Canada, 19961997. Diabetes Care 2004; 27: 407-14.

15. Clarke PM, Gray AM, Briggs A, Farmer AJ, Fenn P, Stevens RJ, Matthews DR, Stratton IM, Holman RR; UK Prospective Diabetes Study (UKDPS) Group.A model to estimate the lifetime health outcomes of patients with type 2 diabetes: the United Kingdom Prospective Diabetes Study (UKPDS) Outcomes Model (UKPDS no. 68). Diabetologia 2004; 47: 1747-59. Epub 2004 Oct 27.

16. Caro JJ, Ishak KJ, Migliaccio-Walle K. Estimating survival for costeffectiveness analyses: a case study in atherothrombosis. Value in Health 2004; 7: 627-35.

17. Rovira J, Antoñanzas F. Economic analysis of health technologies and programmes. A Spanish proposal for methodological standardisation. Pharmacoeconomics 1995; 8: 245-52.
18. Canadian Coordinating Office for Health Technology Assessment. Guideline for economic evaluation of pharmaceuticals: Canada. Ottawa: Canadian Coordinating Office for Health Technology Assessment (CCOHTA), 1997.

19. Weinstein MC, O’Brien B, Hornberger J, Jackson J, Johannesson M, $\mathrm{McCabe} \mathrm{C}$, et al. Principles of good practice for decision analytic modeling in health-care evaluation: report of the ISPOR Task Force on good research practices - modeling studies. Value in Health 2003; 6: 9-17.

20. Base de datos de medicamentos. Consejo General de Colegios Oficiales de Farmacéuticos. Disponible en URL: http://www.portalfarma.com/home.nsf (consulta: junio, 2005).

21. Gisbert R, Brosa M. Base de datos de costes sanitarios. Versión 2.2. Barcelona: Soikos, 2005.

22. Tappenden P, Chilcott JB, Eggington S, Oakley J, McCabe C. Methods for expected value of information analysis in complex health economic models: developments on the health economics of interferon-a and glatiramer acetate for multiple sclerosis. Health Technol Assess 2004; 8: 1-92.

23. Ortún Rubio V. Clínica y gestión. Med Clin (Barc) 1995; 104: 298-300.

24. Del Llano J, Oliva J. Medicina coste-efectiva y medicina basada en la evidencia: su impacto en el proceso de decisiones clínicas. Med Clin (Barc) 2000; 114 (Supl. 3): 34-41.

25. Soto Álvarez, J. Valor terapéutico añadido de los medicamentos: ¿qué es, cómo se evalúa y cuál debería ser su papel en política farmacéutica? An Med Interna (Madrid) 2005; 22: 39-42.

26. Lee JT, Sánchez LA. Interpretation of cost-effectiveness and soundness of economic evaluations in the pharmacy literature. Am J Hosp Pharm 1991; 48: 2622-7.

27. Laupacis A, Fenny D, Detsky A, Tugwell PX. How attractive does a new technology have to be to warrant adoption and utilization? Tentative guidelines for using clinical and economic evaluations. Can Med Assoc J 1992; 146: 473-81.

28. Sacristán JA, Oliva J, Del Llano J, Prieto L, Pinto JL. ¿Qué es una tecnología sanitaria eficiente en España? Gac Sanit 2002; 16: 334-43. 